\title{
$\frac{1}{1682}$
}

Sammlung Metzler Band 224 
Sonja Hilzinger

\section{Christa Wolf}

J.B. Metzlersche Verlagsbuchhandlung

Stuttgart 
CIP-Kurztitelaufnahme der Deutschen Bibliothek

Hilzinger, Sonja:

Christa Wolf / Sonja Hilzinger.

-Stuttgart: Metzler, 1986.

(Sammlung Metzler; M 224)

NE: GT

\section{ISSN 0558-3667}

ISBN 978-3-476-10224-9

ISBN 978-3-476-03928-6 (eBook)

DOI 10.1007/978-3-476-03928-6

\section{224}

(c) Springer-Verlag GmbH Deutschland 1986

Ursprünglich erschienen bei J. B. Metzlersche Verlagsbuchhundlung und Carl Ernst Poeschel Verlag GmbH in Stuttgart 1986 


\section{INHALT}

Vorbemerkung $\ldots \ldots \ldots \ldots \ldots \ldots \ldots \ldots \ldots \ldots \ldots$, VIII

Günter de Bruyn: Fragment eines Frauenporträts ....... 1

1. Christa Wolfs Weg von der Literaturkritikerin

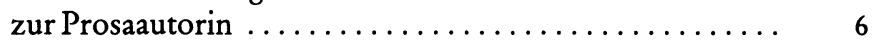

Die Kritikerin ............................ 6

*Moskauer Novelle ........................... 9

-Dergeteilte Himmel ....................... 16

2. "... zu erzählen, das heißt: wahrheitsgetreu zu erfinden auf Grund eigener Erfahrung"

Christa Wolfs neues erzählerisches Selbstverständnis .... 28

*Juninachmittag ........................... 28

*Nacbdenken über Christa T. . ................. 32

Entstehungs- und Rezeptionszusammenhang 32 - Von der Schwierigkeit des Ich-Sagens 33 - Lesen und Schreiben 36-Die Suche nach Heimat 40 - Erzählerisches Verfahren 42

*Lesen und Schreiben* ..........................

Entwurf einer Prosatheorie 47 - Prosa als Experiment 51 - Bezugnahme auf Anna Seghers 53

3. Unwahrscheinliche Geschichten ............... 56

Unwahrscheinlichkeit als Mittel der Verfremdung ......... 56

*Unter den Linden . . . . . . . . . . . . ........... 58

Die erste Geschichte 59-Die zweite Geschichte 60-Die dritte Geschichte 62-Die Weiterführung der ersten Geschichte 63-Die Fundierung der Erzähltechnik in romantischer Tradition 66 *Neue Lebensansichten eines Katers . . . . . . . . . . . . . . . . . 68 *Kleiner Ausflug nach H. . . . . . . . . . . . . . . . 70 ${ }{ }_{\text {Selbstversuch } \ldots \ldots \ldots \ldots \ldots \ldots \ldots \ldots \ldots \ldots \ldots \ldots} 71$

4. Die Filmerzählung $»$ Till Eulenspiegel $\ldots \ldots \ldots \ldots \ldots \ldots \quad 77$

Die feudale Ordnung am Vorabend der frühbürgerlichen Revolution ............................. 77

Die Funktion des Narren. . . . . . . . . . . . . . . . 80

Zeitinterpretation in historischem Gewand $\ldots \ldots \ldots \ldots \ldots . \quad 88$

5. Die Aufarbeitung von Zeitgeschichte am Beispiel der persönlichen Biographie: $»$ Kindheitsmuster * ...... 91

Faschismus-Bewältigung in der DDR-Literatur . . . . . . . 91

Die Erzähler-Figur als Zentrum der Struktur . . . . . . . . . 94

Die erzählerische Gestaltung als Versinnlichung von Geschichte . . 102 
6. Erneuerung romantischer Traditionsbezüge $\ldots \ldots \ldots \ldots \quad 106$

Die Folgen der Biermann-Ausbürgerung . . . . . . . . 106

Die politische Situation um $1800 \ldots \ldots \ldots \ldots \ldots \ldots \ldots . \quad 109$

Neubestimmung der Funktion von Literatur . . . . . . . . . . . . 109

Alternativen in der frühen Romantik . . . . . . . . . . . . . . 110

Die Rolle der Romantikerinnen ................... 111

Die neue Literatur der Romantikerinnen .............. 114

Die Bedeutung der romantischen Traditionswahl ......... 117

Darstellung des erzählerischen Verfahrens in $*$ Kein Ort.

Nirgends .............................. 120

Die Alternative: Berührung . . . . . . . . . . . . . . 123

Die Funktion der Erzählerfigur . . . . . . . . . . . . 128

7. "Literatur heute muß Friedensforschung sein."

„Voraussetzungen einer Erzäblung: Kassandra "...... 130

Der Rückgriff auf den Mythos ................... 130

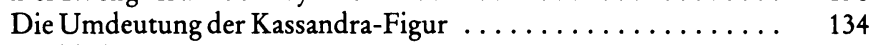

Weibliche Utopie? ............................ 139

Weibliches Schreiben?....................... 143

Abkürzungsverzeichnis .................... 147

Bibliographie $\ldots \ldots \ldots \ldots \ldots \ldots \ldots \ldots \ldots \ldots \ldots \ldots \ldots \ldots \ldots$

Daten zu Leben und Werk $\ldots \ldots \ldots \ldots \ldots \ldots \ldots \ldots \ldots \ldots$

Namenregister ........................ 190 


\section{VORBEMERKUNG}

Nicht erst seit dem Erscheinen ihrer jüngsten Erzählung »Kassandra « ist die DDR-Autorin Christa Wolf in den Blickpunkt affirmativer, vereinnahmender oder kritischer Rezeption durch eine interessierte bundesrepublikanische Öffentlichkeit, durch Friedens- und Frauenbewegung gerückt.

Die westliche Rezeption ihrer Prosa und Essayistik seit nahezu zwanzig Jahren ist jedoch dort von mangelnder Differenziertheit und der Gefahr einseitiger oder mißverständlicher Interpretation bedroht, wo Christa Wolfs kritisch-loyale Haltung zur DDR, dem Land, in dem sie lebt und arbeitet, zu sehr aus dem Blickfeld gerät.

Christa Wolfs Prosa und Essayistik ist kaum angemessen zu verstehen und einzuordnen, löst man sie aus ihrem Produktionszusammenhang. Die fortwährende, engagierte Auseinandersetzung mit Fortschritt und Rückschlägen sozialistischer Entwicklung in der DDR hat die schriftstellerische Arbeit Christa Wolfs bis heute nachhaltig geprägt. Diese Grundlage ihrer Prosa und Essayistik versucht der vorliegende Band zu erfassen und darzustellen. Im Unterschied zu anderen Gesamtinterpretationen des Werks Christa Wolfs finden daher zwei Phasen besondere Beachtung. Dies ist zum einen die komplizierte Entwicklung Christa Wolfs von der Literaturkritikerin zu einer Prosaautorin mit einem eigenständigen prosatheoretischen Entwurf in den sechziger Jahren; zum anderen die konsequente Bearbeitung ihres Verhältnisses zur DDR nach der Ausweisung Wolf Biermanns in ihren literarischen Arbeiten Ende der siebziger Jahre.

Die biographische Zurückhaltung Christa Wolfs - darin ähnelt sie der anderen großen Autorin der DDR, Anna Seghers - verweist nachdrücklich auf ihr Werk, das in bemerkenswerter Offenheit die Spuren, welche die Auseinandersetzung mit der Realität in die Psyche eingräbt, hervortreten läßt. Diesem Befund trägt der vorliegende Band Rechnung in einer Darstellungsweise, die stärker einfühlend und nachvollziehend als wertend vorgeht und psychologische, gesellschaftliche und ästhetische Dimensionen gleichermaßen zu erfassen sucht.

Die Kapitaleinteilung folgt den Entstehungszeiten der Arbeiten Christa Wolfs und rekonstruiert sowohl deren subjektive Produktionsbedingungen als auch kulturpolitischen Voraussetzungen. Die Bibliographie ist den einzelnen Kapiteln zugeordnet und gibt fundierteren Arbeiten der Sekundärliteratur den Vorzug gegenüber Rezensionen. Eine Übersicht über die Daten von Leben und Werk Christa Wolfs schließt den Band ab. 


\section{GÜNTER DE BruYN ÜBer Christa Wolf \\ Fragment EINES FraUENPORTRÄTS}

\section{Maler möchte ich sein!}

Das denkt einer, dem Schreiben schwerfällt in diesem Fall. Weil seine Mittel dem Gegenstand nicht angemessen sind. Malen, denkt er, wäre leichter. Man hielte sich an das, was man sieht, und vertraute darauf, daß man damit auch träfe, was man weiß und fühlt, wenn man vor dieses Gesicht gestellt ist. Das Haar ist dunkel. Die Brauen, weil auch dunkel, sind deutlich herausgehoben; tief angesetzt, beschatten sie Augen schwer erkennbarer Färbung. Grau, grün, blau, graugrün, graublau? Bezeichnend die Nase: schon vom Ansatz her Tendenzen zur Breite. Wenn der Pinsel ihre Linien trifft, ist äußere Ähnlichkeit schon garantiert. Passend dazu dann die Flächen, groß, ausgedehnt, die Stirn, die Wangen. Diesem Gesicht kann man, denkt er, wenn man Bescheid weiß, ansehen, wo es herkommt. Nicht gerade Landsberg an der Warthe, aber doch die Richtung. So, wie man sich einbilden konnte, Bobrowski Ostpreußen anzusehen. $\mathrm{Da}$ es so selten ist, lohnt es sich, darauf hinzuweisen, wenn Schablonen mal passen.

Lachen darf dieses Gesicht nicht, denkt er, obwohl er es auch so schon gesehen hat, gar nicht selten. Lächeln ginge, aber gut wäre es nicht. Ganz und gar nicht vorstellbar ist Zynismus. Eher könnte es da böse aussehen. Es gibt Leute, die kennen es so, vergessen es nicht und würden es so malen. Möglich wäre auch das, aber vom Wesentlichen böte es nichts, gar nichts.

Wärme? Melancholie? Güte?

Er macht den Versuch, das Gesicht in Ruhestellung zu bringen, es sich entspannen zu lassen, von Gemütsbewegungen frei zu halten, um nur das herauslösen zu können, was drin ist, von Natur aus, im Gesicht an sich. Und sofort hat er es: Nachdenklichkeit. Selbstverständlich, was hätte näher glegen! Schließlich hat die Frau ihren Stil. Doch welche Nachdenklichkeit ist ohne Melancholie? Und ohne Unruhe?

Aber das ist schon mehr, als sich aus diesem Gesicht herauslesen läßt. Er ist schon bei dem, was er weiß. Sehen und Wissen gehen ineinander über, aber überdecken sich nicht. Wie jeder, der ihre Bücher kennt, weiß er vieles von dem, was das in sich ruhende Gesicht verbirgt.

Ein Maler möchte er sein, der das könnte: hinter nachdenklicher Ruhe Qual sichtbar machen, hinter Weichheit Standhaftigkeit, hinter Schwere Sensibilität. Doch wie stellt man ein Gesicht dar, das 
Verwundbarkeit eher verdeckt als spiegelt? Mit welcher Farbe malt man Aufrichtigkeit?

Zum Glück ist er kein Maler. Nicht Farben braucht er, sondern Worte. In Worten muß er darstellen, wie das vorgestellte Bild aussieht. Um Vorstellung aufzufrischen, fährt er zu ihr. Er will sie ansehen, anders als sonst, als Beobachter, mit dem Bewußtsein, das, was er sieht, darstellen zu müssen. Während er sie ansieht, muß er gedanklich schon Skizzen entwerfen zum geplanten Porträt. Er muß so fragen, daß ihre Antworten enthüllen, was er noch nicht weiß. In sie hineinzusehen muß er versuchen - aber nicht wie in einen Spiegel. Er weiß, daß man nur schwer der Gefahr entgeht, Porträts zu Selbstporträts zu machen. Schon mit der Auswahl seines Objekts legt der Porträtist sich fest - auf sich selbst. Als wesentlich sieht er an, was ihm selbst wichtig ist. Nicht was ist, stellt er dar, sondern was er kann. Um dieser Gefahr zu entgehen, muß er versuchen, sich neben sich zu stellen, mehr und anderes zu sehen, als er gewöhnlich sieht, von anderer Richtung, von anderem Standort aus zu sehen. Das alles nimmt er sich vor.

Die Lage ihres Wohnorts läßt vermuten, daß sie auf Besucher wenig Wert legt. Oder nur auf solche, die keine Mühe scheuen. Er muß vier Verkehrsmittel benutzen, Straßenbahn, S-Bahn, Dampfbahn, ansehen, anders als sonst, als Beobachter, mit dem Bewußtsein, das, Bus. Auf der Fahrt hätte er Zeit, sich die Fragen, die er ihr stellen welche stellt und nicht beantworten kann. Wie hätte er sich nach der Lektüre ihrer Bücher, kennte er die Autorin nicht, ihr Gesicht vorgestellt? Wie das der Seghers, der Huch, der George Sand, der Bettina oder der Ebner-Eschenbach? Oder anders gefragt: Las er aus ihrem Gesicht wirklich etwas heraus oder nicht vielmehr hinein? Welchen Eindruck hatte er denn, als er es vor fünfzehn, sechzehn Jahren zum erstenmal sah, höhnt er. Damals war sie Angestellte des Schriftstellerverbandes, er Angestellter eines Bibliotheksinstituts. Einem Kongreß hatte bibliographisches Material vorgelegt werden sollen. Er war zu ihr geschickt worden, um Rat und Hilfe anzubieten. Damals hatte er mit dem Hochmut des Spezialisten Widersprüche zwischen Schein und Sein entdeckt: die redet von Wissenschaftlichkeit und weiß nicht, wie man Register der Nationalbibliographie benutzt, gibt sich arrogant, um Unsicherheit $\mathrm{zu}$ verdecken. Heute gibt er, aus der Erinnerung rekonstruiert, seinen Eindruck so wieder: ein Gesicht, das sich noch nicht wahrhaben wollte. Auch an einen ihrer Artikel aus dieser Zeit erinnert er sich. In welcher Welt lebt dieses Mädchen, dachte er, daß es eines Krankenhausaufenthalts bedarf, um in ihr Entsetzen über die verbreitete Sucht nach Trivialliteratur zu erregen. 
Vielleicht kam damals die Nachdenklichkeit in dieses Gesicht, begann die Entdeckung dessen, was sie war, begann, mit dem Abbau von Illusionen, mit Erfahrungen und Enttäuschungen, die Entdekkung der Wirklichkeit, die sie zum Schriftsteller machte. Die Moskauer Novelle zeigt noch wenig davon, doch ist ein Stil schon da, ein Anspruch wird angemeldet, ein Bild vom Menschen formuliert, das auf das hindeutet, was danach kommt: der geteilte Himmel, die Christa T.

Obwohl Winter ist, denkt er an ihre kleine, großartige Geschichte vom Juninachmittag, als er ihre Wohnung betritt. Das Kind, heute schon so groß wie damals die Tochter, empfängt ihn. Bis die Eltern ohne Hast von ihren Arbeitsplätzen kommen, kann er mühelos mit ihm plaudern. Nicht weil es wie ein Erwachsener wirkt, sondern weil es mit Selbstverständlichkeit Kind ist. Jeder ist hier mit Selbstverständlichkeit er selbst.

Es gibt Familien, die durch Überzahl und Geschlossenheit Besucher einschüchtern, andere, die durch den Aufwand, den sie ihretwegen treiben, schlechtes Gewissen bei ihnen erzeugen. Hier, wo man Formationsbildung nicht nötig hat, weil jede Individualität die andere gelten läßt, braucht man die eigene nicht $\mathrm{zu}$ unterdrücken. $\mathrm{Da}$ nicht viel Wesens von einem gemacht wird, empfindet man sich nicht als störend. So märchenhaft intakte Familien gibt es also, kann er gerade noch denken, da gehört er schon fast dazu. Da noch Essensvorbereitung erforderlich ist, geht er mit in die Küche, versucht zu helfen, da doch alle helfen, darf aber auch in der Ecke sitzen und reden. Denn er wird gefragt. Nach Arbeiten, über die er vor Jahren einmal gesprochen hat, nach Freunden, Reisen, Krankheiten, nach Meinungen über dies und das, auch über Kunst.

Erst antwortet er kurz, wie er es gewohnt ist durch Umgang mit eiligen, ungeduldigen Leuten, die nur aus Höflichkeit fragen und das Ende der Antwort kaum abwarten können, weil sie von sich reden wollen. Dann aber, wie immer aufs neue überrascht von dem wirklichen Interesse, aus dem hier gefragt wird, beginnt er zu erzählen, genauer, ausführlicher, auch intimer, als er das sonst kann.

Sich verstehen, sich lieben, zusammengehören heißt hier nicht unbedingt: einer Meinung sein. So steht er nie allein gegen zwei, drei, vier. So ist es nicht schwer, zu sagen, was er denkt, Kritik, auch scharfe, zu ertragen. Auf Unverständnis stoßen würde hier nur Besserwisserei und Schauspielerei. Es ist angenehm, nie der Versuchung ausgesetzt zu sein, eine falsche Rolle zu spielen.

Auch vom Essen versteht man viel in dieser Familie. Er lobt, weiß aber, daß er es auch bleibenlassen könnte. Er raucht, ohne sich in diesem Kreis von Nichtrauchern verbrecherisch vorzukommen. 
Wenn er Mohammedaner wäre und in der Zeit seines Besuches Gebete verrichten müßte, würden vielleicht die Töchter mit ihm diskutieren wollen, auf Ablehnung oder Hohn stoßen würde er nicht. Ursprung von Intoleranz ist Angst. Man mißachtet, unterdrückt andere Meinungen, weil man eigene für gefährdet hält. Unsicher der eigenen Wahrheit, riegelt man sich gegen fremde ab. Abgeschlossene Wahrheit aber stockt, verfault, verkommt.

Wer sich, wie diese Frau, einiger Dinge sicher ist, bleibt offen für Neues, ist nicht am Ende, sondern auf der Suche. Das verleiht ihr Autorität, die aber für andere nichts Bedrückendes hat. Sie gibt mit der Geste des Nehmens. Sie fordert auf, ihr beim Suchen behilflich zu sein, hilft dabei aber dem anderen, da sie weit vorn sucht.

Der Besucher, anstatt, sie beobachtend, seiner Arbeit nachzugehen, fragt sich, ständig dazu aufgefordert, über sich selbst nachzudenken, warum er sich hier jünger vorkommt, als er ist. Alle drei gehören sie einer Generation an. Er ist sogar ein paar Jahre älter als sie. Früher, in dem für beide wichtigen Jahr 1945, hätte das eventuell eine Rolle gespielt. Der Unterschied zwischen 17 und 20 ist größer als der zwischen 42 und 45 . Wäre er ihr in dem mecklenburgischen Dorf etwa, in das sie der Krieg verschlagen hatte, begegnet, hätte er vielleicht annehmen können, mehr erfahren, mehr erlebt, mehr gewußt zu haben. Inzwischen aber ist das anders geworden. Sie hat mehr geglaubt, tiefer gedacht. Was bei ihm Zuneigung war, war bei ihr Begeisterung. Wo er zweifelte, verzweifelte sie. "Die verpaßten Erschütterungen sind nicht nachholbar", kann man bei ihr lesen. "Eine Welt, die nicht zur rechten Zeit verzaubert und dunkel war, wird, wenn das Wissen wächst, nicht klar, sondern dürr. Fad und unfruchtbar sind die Wunder, die man seziert, ehe man sie glauben durfte."

Das zielt, von Literaturerfahrung ausgehend, auf Lebenserfahrung, auf Fülle des Lebens, auf Reichtum menschlicher Existenz. Aber verglichen mit diesem Ziel, ist ihr Werk noch klein. Sie ist sich dessen bewußt. Ihre Unzufriedenheit mit sich läßt noch Großes erwarten.

Vielleicht haben Natur und Geschichte, denkt der Besucher, die Frauen dazu prädestiniert, Humanität zu bewahren. Durch Unterdrückung der Erziehung zum Unterdrücken, zur Brutalität, zum Töten entgangen, kann ihre Befreiung zur Befreiung der Menschlichkeit werden. Aristophanes, Goethe, Hölderlin haben davon gewußt, und geahnt haben es alle Schlagetots, die das Wort Humanität nie ohne den Zusatz Duselei verwendeten und Güte als weibisch beschimpften. Die Empfehlung des vom Willen zur 
Macht Besessenen, zum Weibe die Peitsche mitzunehmen, weist auf die Gefahr, die ihm von dorther droht.

Mancher Mann begegnet ihr, indem er an Frauen anerkennt - was Nutzen für Männer hat. Ihre Schönheit zum Beispiel. Da wird eine Frau ihrer Arbeiten wegen in einen erlauchten Kreis aufgenommen, und der Redner läßt sein Lob dahin gipfeln, daß sie Männeraugen einen erfreulichen Anblick bietet. Der Besucher erinnert sich, wie das Gesicht, das er porträtieren soll, dabei aussah. Verwundet. Man mußte Angst um sie haben. Schimpfworte, die sie in solchen Fällen parat hat, verdecken nur, schützen aber nicht. Wenn sie sie benutzt, zuckt er zusammen, hört weg, weil sie das Bild beschädigen könnten, das er von ihr hat.

Vielleicht ist es ein ungenaues, vielleicht ein falsches Bild. Verehrung neigt zu Vereinfachung. Deshalb eignet er sich wohl nicht zu ihrem Porträtisten. Er fährt zu ihr, um sie zu beobachten, fühlt sich wohl, statt zu arbeiten, und als er sich verabschiedet, merkt er, daß sie ihn dazu gebracht hat, mehr über sich selbst nachzudenken als über sie. Zu dem fremden Standort, den er hatte einnehmen wollen, hat er nicht kommen können. Vor dem Gesicht, das er erforschen wollte, stand er wie vor dem eignen Gewissen. Er kann verstehen, daß mancher das nicht mag. Der wehrt sich dann mit Anschuldigungen - und charakterisiert sich selbst.

Nicht einmal über ihre Augenfarbe weiß er mehr als vorher. Das Porträt wird unvollständig bleiben. Aber welches ist das nicht. Und schließlich gibt es ihre Bücher.

(Aus: Liebes- und andere Erklärungen. Schriftsteller über Schriftsteller, hrsg. von Annie Voigtländer, Berlin/Weimar: Aufbau Verlag 1972, S. 410-416) 